\title{
Effect of Rutin on Diabetic Auditory Neuropathy in an Experimental Rat Model
}

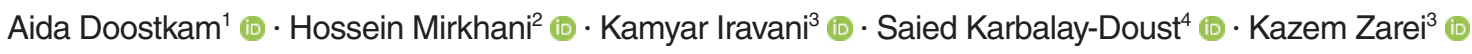 \\ ${ }^{1}$ Department of Pharmacology, School of Medicine, Shiraz Nephro-Urology Research Center, ${ }^{2}$ Department of Pharmacology, Medicinal and \\ Natural Products Chemistry Research Center, School of Medicine, ${ }^{3}$ Department of Otolaryngology, Otolaryngology Research Center, and \\ ${ }^{4}$ Department of Anatomy, Histomorphometry and Stereology Research Center, School of Medicine,
}

Shiraz University of Medical Sciences, Shiraz, Iran

Objectives. Diabetic auditory neuropathy is a common complication of diabetes mellitus that has a major impact on patients' quality of life. In this study, we assessed the efficacy of rutin in treating diabetic auditory neuropathy in an experimental rat model.

Methods. Forty Sprague-Dawley rats were randomly assigned to the following groups: group 1, control; group 2, diabetic rats; and groups 3-5, rats treated with rutin (at doses of 50,100 , and $150 \mathrm{mg} / \mathrm{kg}$, respectively). We used auditory brain stem response, stereology of the spiral ganglion, and measurements of superoxide dismutase (SOD) and malondialdehyde (MDA) to evaluate the effects of treatment.

Results. Significant improvements in auditory neuropathy were observed in the rutin-treated groups in comparison with the diabetic group $(P<0.05)$. Auditory threshold, wave latency, wave morphology, the volume and number of neurons in the spiral ganglion, and SOD and MDA activity showed improvements following treatment.

Conclusion. Rutin shows promise as a treatment modality for diabetic auditory neuropathy, but more trials are warranted for its clinical application.

Keywords. Diabetic Neuropathy; Rutin; Histology; Auditory Brainstem Response; Animal Model

\section{INTRODUCTION}

Diabetes mellitus (DM) is a metabolic disease that affects multiple organs throughout the body. Diabetes imposes a high burden in terms of mortality and morbidity, primarily due to vascular and nervous system (peripheral and central) involvement. Diabetic neuropathy involves sensory, motor, and autonomic nerves $[1,2]$. Metabolic aberrations in neuropathy are mainly the result of oxidative stress and the polyol pathway. Hyperglycemia upregulates the polyol pathway, aldose reductase activity, and sor-

\footnotetext{
- Received December 18, 2019

Revised March 26, 2020

Accepted May 5, 2020

- Corresponding author: Kamyar Iravani

Department of Otolaryngology, Otolaryngology Research Center,

Shiraz University of Medical Sciences, Khalili Hospital, Khalili St.,

Shiraz 71936-16641, Iran

Tel: +98-9171910769, Fax: +98-7136291478

E-mail: iravanika@sums.ac.ir
}

bitol production. Hyperglycemia also increases the formation of advanced glycation end-products, activates protein kinase $\mathrm{C}$, poly (adenosine diphosphate-ribose) polymerase, and the hexosamine pathway, and causes changes in neurotrophic factors. These mechanisms all result in increased levels of free radicals, especially reactive oxygen species (ROS), in tissues throughout the body $[3,4]$. In addition to radical species, hyperlipidemia and persistent hemoglobin glycosylation damage the vascular endothelium. Endothelial damage causes basement membrane thickening and cellular proliferation, thereby shrinking the vascular lumen [5]. Neuropathy in DM primarily occurs to damage to feeding vessels, as the effects of ischemia on nerves include the demyelination and degeneration of neural fibers. Microcirculation disorders also affect the auditory system through structural and functional changes. Auditory neuropathy clinically presents with hearing loss, tinnitus, and dizziness, which have a major effect on quality of life [6,7]. Despite our knowledge of the mechanisms underlying diabetic neuropathy, little is known

Copyright @ 2021 by Korean Society of Otorhinolaryngology-Head and Neck Surgery.

This is an open-access article distributed under the terms of the Creative Commons Attribution Non-Commercial License (https://creativecommons.org/licenses/by-nc/4.0)

which permits unrestricted non-commercial use, distribution, and reproduction in any medium, provided the original work is properly cited. 
about its prevention and treatment. Many agents, such as benfotiamine (a vitamin B1 derivative) [8,9], alpha-lipoic acid [10], folic acid [11], and ruboxistaurin (a protein kinase C-beta inhibitor) [9] have been investigated in experimental and clinical trials to determine whether they reduce the rate and severity of neuropathy, but none has led to definitive results; thus, further studies are needed to find an effective way to manage diabetic neuropathy $[8,9]$.

Rutin, also called rutoside, is a bioflavonoid formed by the flavonol quercetin and the disaccharide rutinose. This glycoside is found in many plants and fruits, including citrus fruits, apple, and buckwheat $[12,13]$. Rutin is known to have anti-inflammatory, antiproliferative, and antioxidant activity, the latter of which it exerts by scavenging ROS. Reducing levels of ROS can decrease oxidative stress in neurons, thereby controlling neuropathy (including auditory neuropathy) in patients with diabetes [14-16].

The auditory brain stem response (ABR), which is measured by evoked potential recording, is one the best tools to evaluate auditory neuropathy. It reflects electrical activity in the auditory nerve and its brainstem connections. The electrical activity recorded after stimulation includes a series of five to seven vertexpositive waves, each of which is considered to correspond to a specific site in the auditory pathway. Although the localization is not fully clear, it is believed that waves I, II, III, IV, and V correlate with the activity of the auditory nerve, cochlear nucleus, superior olivary complex, lateral lemniscus, and inferior colliculus, respectively $[7,17,18]$. DM has been shown to affect the microvasculature of the cochlea, and the resultant ischemia causes morphological changes in the stria vascularis, sensory cells, and spiral ganglion $[6,19]$. The spiral ganglion is a group of neurons in the modiolus, the dendrites of which primarily synapse with inner hair cells and to a lesser extent with outer hair cells. Axons of ganglionic cells comprise the auditory nerve [2]. We used stereology for an exact evaluation of spiral ganglion structure. In this method, a three-dimensional reconstruction of tissues was evaluated and interpreted quantitatively with minimal bias. Using the stereological technique, the volume and number of neural cells in the spiral ganglion were assessed in diabetic rats and after treatment with rutin.

Superoxide dismutase (SOD) is a group of enzymes that catalyze the transformation of superoxide radicals into oxygen and

\section{H I G G H L L I}

- Rutin has significant therapeutic effects on auditory neuropathy.

- ABR recordings were improved following rutin therapy in diabetic rats.

- Stereologic studies showed improvement in number and volume of spiral ganglions after rutin treatment. hydrogen peroxide $\left(\mathrm{H}_{2} \mathrm{O}_{2}\right)$ [20]. These enzymes exert antioxidant activity that plays an important role in controlling oxidative stress. In some studies, SOD has been measured as a biomarker to evaluate neural damage in neuropathy [21]. Malondialdehyde (MDA; $\mathrm{C}_{3} \mathrm{H}_{4} \mathrm{O}_{2}$ ) is a product of lipid peroxidation of polyunsaturated fatty acids. It has been used as a biomarker to measure levels of oxidative stress in many experimental and clinical studies [22,23]. In our study, we used ABR as a tool for functional evaluation, the stereological technique to evaluate the volume and number of neurons in the spiral ganglion, and MDA and SOD as biomarkers to evaluate the efficacy of rutin on diabetic auditory neuropathy.

\section{MATERIALS AND METHODS}

\section{Animal preparation}

Forty Sprague-Dawley male rats (250-300 g) were used in this study. The rats were housed individually in animal laboratory under controlled conditions of temperature $\left(24^{\circ} \mathrm{C} \pm 2^{\circ} \mathrm{C}\right)$, humidity, 12 hours light/dark cycle per day, free access to food and water. Rats were divided randomly into five groups $(n=8$ per group) as follows: nondiabetic rats with distilled water (normal), diabetic rats with distilled water without rutin treatment (DM), diabetic rats with rutin $(50 \mathrm{mg} / \mathrm{kg} / \mathrm{day})$ treated $(\mathrm{DM}+\mathrm{R} 50)$, diabetic rats with rutin $(100 \mathrm{mg} / \mathrm{kg} /$ day $)$ treated $(\mathrm{DM}+\mathrm{R} 100)$, and diabetic rats with rutin $(150 \mathrm{mg} / \mathrm{kg} /$ day) treated (DM+R150). Diabetes was induced by a single intraperitoneal injection of streptozotocin (STZ; 60 mg/kg, Sigma-Aldrich, St. Louis, MO, USA) dissolved in cold citrate buffer. The fasting blood sugar was tested 3 days after STZ injection and rats with blood sugar greater than $300 \mathrm{mg} / \mathrm{dL}$ were considered as diabetic [24].

Rutin (Sigma-Aldrich) powder was dissolved in distilled water and administered after confirmed diabetes (3 days after STZ injection), using the above mentioned doses through daily gastric gavage for 8 weeks. After 8 weeks of treatment, rats were prepared for auditory function testing by ABR and histological studies. The study protocols were approved by the local Ethics Committee of Shiraz University of Medical Sciences (No. IR.SUMS. REC.1397.459).

\section{ABR recording}

Anesthesia was done with Xylazine $(10 \mathrm{mg} / \mathrm{kg})$ and Ketamine (75-100 mg/kg) via intraperitoneal injection [25]. To prevent hypothermia, we used heat lamp and thermal blanket. In the ABR recording, active electrode (+) (ECG Ambu Blue Sensor, Penang, Malaysia) was attached to the forehead and the reference electrode $(-)$ to the post auricular area of right and left ears. Ground electrode was attached to the dorsal neck area. Evoked potentials were obtained by intra acoustic EP25 (Copenhagen, Denmark) system with otoaccess software (Middelfart, Denmark) after delivering clicks to the right ear via insert- 
ed earphones. At least 700 stimuli at a rate of $11.1 \mathrm{~Hz}$ were delivered to right ear. Electrode impedance was below $3 \mathrm{~K} \Omega$. ABR recordings were obtained with band pass filter of $100-3,000 \mathrm{~Hz}$ and time window of $10 \mathrm{~ms}$ [26]. Evoked potentials were evaluated according to the following parameters: auditory threshold, the absolute latencies of wave's II and V and wave morphology. Auditory threshold was determined in $5 \mathrm{~dB}$ steps decreasing from maximum stimulus of $70 \mathrm{~dB}$ until wave forms lost their morphology.

\section{Tissue preparation \& stereological studies}

After dissecting and separating the temporal bone from its neighboring skull bones carefully, it was decalcified with $8 \%$ HCL and 8\% formic acid for 72 hours [27]. All temporal bones were processed and embedded in paraffin, so that the external acoustic meatus was placed on the floor of the block. The blocks were serially sectioned (25- $\mu \mathrm{m}$ thickness) together with the external acoustic meatus axis using a microtome (Microm, Walldorf, Germany). The serial sections of cochlea were selected and separated from the beginning to the end. The mean of $34 \mathrm{sec}$ tions per animal in cochlear slides were obtained and stained with Hematoxylin and Eosin. Then, 8-12 sections were chosen out of the total sections of cochlea in a systematic uniform random method. Stereological methods were used to estimate the volume of the spiral ganglion and the number of neurons in that ganglion. The study was performed by an examiner (SKD) who was blind to the allocation groups.
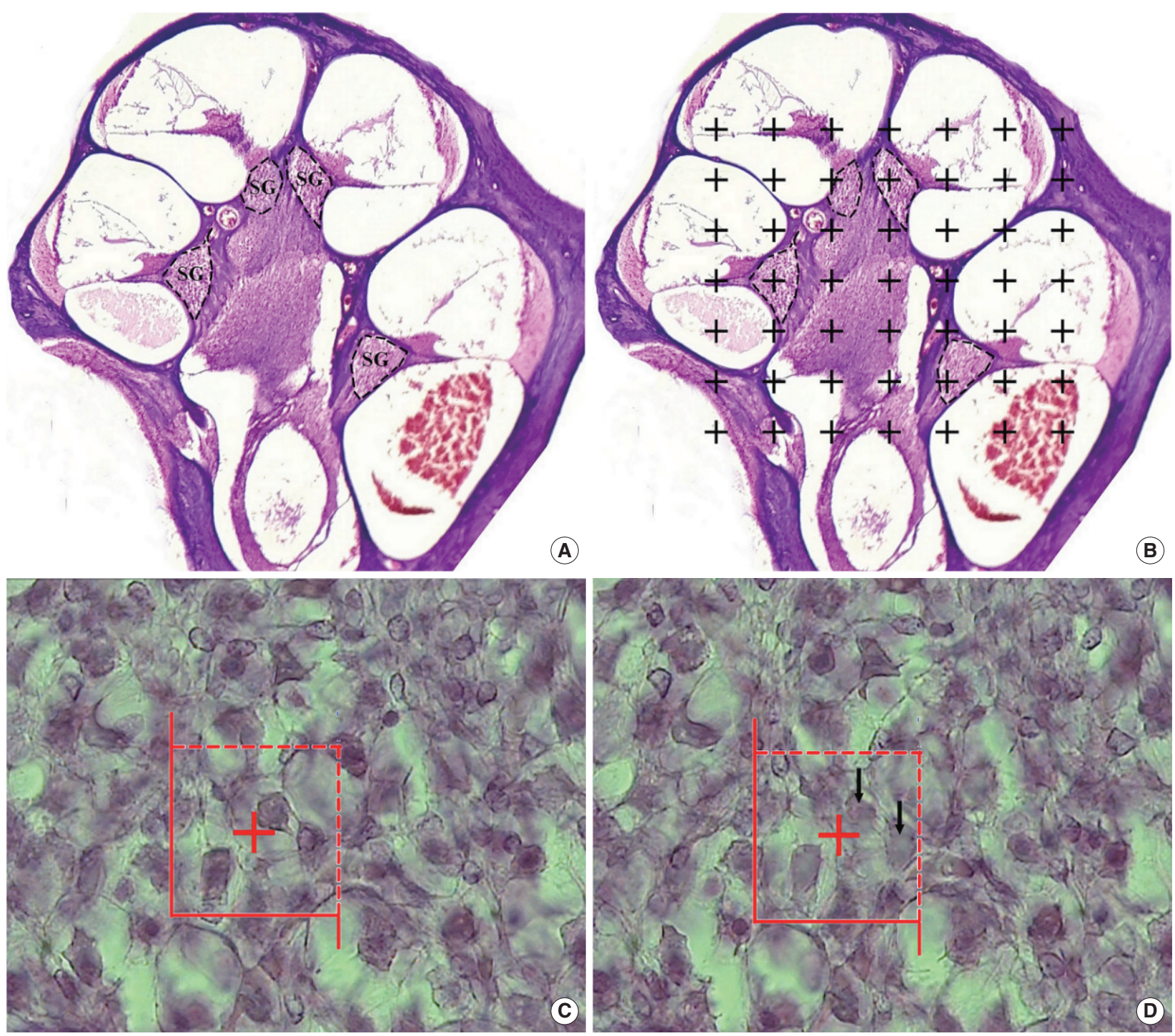

Fig. 1. Use of the stereological technique to estimate the spiral ganglion (SG) volume and cell number. H\&E stain. (A) The location of the SG of the cochlea shown on a histological section. (B) The volume of the SG was estimated using Cavalieri's method and the point-counting method. (C, D) The cell number of the SG was evaluated by using the optical disector method. The SG cells' nuclei were documented as they came into focus throughout scanning by varying the altitude of the disector (arrows). 


\section{Estimation of the spiral ganglion volume}

The live image of each cochlea section was assessed by a microscope linked to a computer. In each section cochlea, boundaries and the borders between spiral ganglion regions were recognized. The total volume of spiral ganglion was assessed on 8-12 sections per animal at the final magnification of 83 with the "Cavalieri's principle based on point count method" [28-30] by the stereology software (StereoLite, SUMS, Shiraz, Iran). The stereological probe (a grid of points) was superimposed over images cochlea. Thus, the areas sections of $\sum \mathrm{A}$ (spiral ganglion) were multiplied by the sampled sections thickness $(\mathrm{T})$. The area was assessed by the stereology software, and the volume of spiral ganglion was assessed by the following formula:

$$
\mathrm{V}=\mathrm{T} \cdot a / p \cdot \sum \mathrm{P}
$$

, where $\mathrm{V}$ is the volume of spiral ganglion; $\mathrm{T}$ is distance between the two selected sections; $a / p$ is area per point; and $\sum \mathrm{P}$ is total points hitting spiral ganglion.
The $a / p$ was $9,289.1 \mu \mathrm{m}^{2}$ and on average 165 points were counted per animal.

\section{Estimating the number of neurons in the spiral ganglion}

The cell bodies of the bipolar neurons of the spiral ganglion were observed in the modiolus of ear, the lemon-like shaped central axis in the cochlea [28]. The number of neurons in each unit volume of the cochlea was estimated by the "optical disector" technique [30-32].

The location of the microscopic fields was selected by moving the stage in $\mathrm{X}$ - and Y-directions at equal distances according to systematic uniform random sampling. Objective lens $(40 \times$, numerical aperture 1.30) was used by oil immersion. An unbiased counting frame consisting of two exclusion lines and two inclusion lines was superimposed on the sections of the cochlea. The focal plane of microscope was moved down in $\mathrm{z}$ direction. A microcator (MT 12; Heidenhain, Traunreut, Germany) was contacted to the microscope to determine the $\mathrm{z}$-axis movement. The guard regions were applied to avoid cutting artifacts that oc-
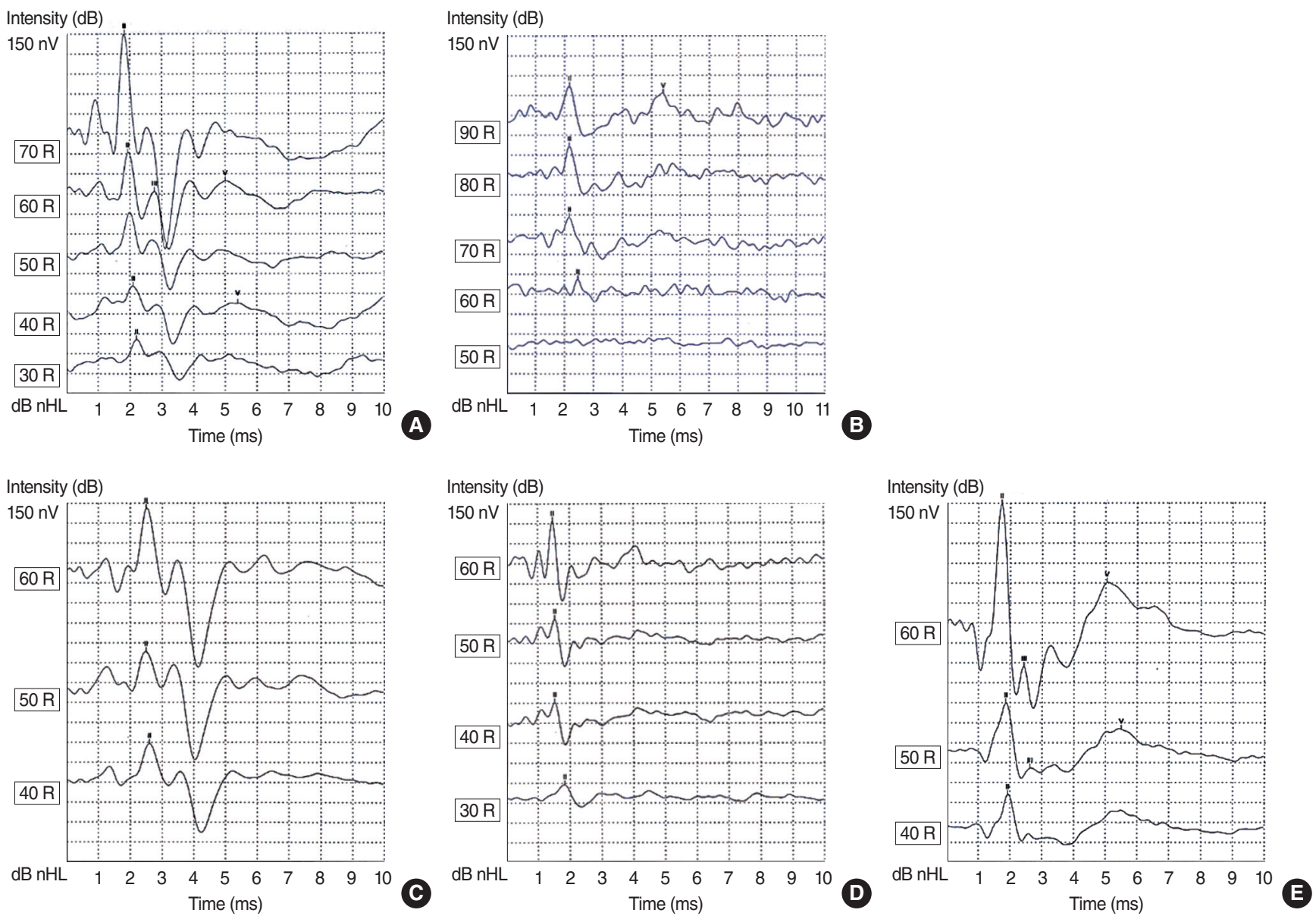

Fig. 2. Auditory brain stem response (ABR) recordings in normal, diabetic, and rutin-treated mice. $A B R$ recordings in the $(A)$ normal, $(B)$ DM, (C) DM+R50, (D) DM+R100, and (E) DM+R150 groups. DM, diabetes mellitus; R50, 50 mg/kg of rutin; R100, 100 mg/kg of rutin; R150, 150 $\mathrm{mg} / \mathrm{kg}$ of rutin. 
curred on the upper and lower surfaces of the histological sections. Each nucleolus cell appears in focus inside the upper (the first $5 \mu \mathrm{m}$ ) or lower guard regions were ignored. The "height of disector" was the interval between the guard regions which was $15 \mu \mathrm{m}$ here. Any nucleolus cell coming into the focus within the subsequent focal sampling plane was chosen if it was placed totally or partly within the counting frame and did not contact the exclusion lines [30-32].

The numerical density of neurons (Nv) was estimated using the following formula:

$$
\mathrm{Nv}=\left[\sum \overline{\mathrm{Q}} /\left[\sum \mathrm{P} \times a(f) \times \mathrm{h}\right]\right] \times[\mathrm{t} / \mathrm{BA}]
$$

Where $\sum \bar{Q}$ was the number of the neuron cell nucleoli coming into focus; $\sum$ P was the sum of the unbiased counting frame in all fields; and $\mathrm{h}$ was the height of disector $(15 \mu \mathrm{m}) ; a(f)$ the counting frame area was $1,082.41\left(32.9 \times 32.9 \mu^{2}\right)$; $t$ was the real average section thickness measured by the microcator; and BA was the block advance of the microtome set at $25 \mu \mathrm{m}$. On the average, 100-150 neuron cells were counted in 23-45 disectors in 8-12 sections per spiral ganglion. The total number of the spiral ganglion neuron was estimated by multiplying the numerical density (Nv) by the $\mathrm{V}$ (spiral ganglion). $\mathrm{N}_{\text {Total }}=\mathrm{Nv} \times \mathrm{V}$ (Fig. 1 ).

\section{Biochemical assays}

SOD measurement in plasma was done after 8 weeks in all normal and case groups, using SOD enzyme-linked immunosorbent assay kit (Cayman, Ann Arbor, MI, USA). MDA assay in plasma was done by reaction with thiobarbituric acid (TBA) and quantification of their combination (MDA-TBA) colorimetrically (532 nm).

\section{Statistical analysis}

All parametric data in our study were expressed as mean for stereological results and mean \pm standard error of the mean for other results. For statistical analysis, Kruskal-Wallis test for nonparametric data such as different forms of wave $\mathrm{V}$ and analysis of variance for parametric data such as latency of wave II, hearing threshold, volume of spiral ganglion, number of spiral ganglion neurons, superoxidase dismutase and MDA were applied. GraphPad Prism 6 software (GraphPad Software Inc., San Diego, CA, USA) was used to compare data. Dunn and Tukey tests were applied for comparisons between groups. The values of $P<0.05$ were considered as statistically significant.

\section{RESULTS}

\section{The effects of rutin on $A B R$}

DM caused significant effect on ABR wave forms, the latency of wave II, the presence of waveV, and the hearing threshold (Fig. 2). The latency of wave II is shown in Fig. 3A.All rutin-treated groups showed improvements (decreases) in wave II latency. In the groups that received 100 and $150 \mathrm{mg} / \mathrm{kg}$ of rutin, the improvement was statistically significant $(P<0.01$ and $P<0.0001$, respectively). The hearing threshold was increased in diabetic rats, but returned to normal limits after rutin treatment $(P<0.05$ and $P<0.001)$ (Fig. $3 B)$. DM also had negative effect on the presence of wave $V$ in ABR recordings. Rutin treatment, especially at a dose of 150 $\mathrm{mg} / \mathrm{kg}$, led to a significant improvement in the presence of wave V $(P<0.05)$ (Table 1).

\section{Histological studies}

Volume of spiral ganglion evaluation

A significant reduction (52.39\%) was found in the total volume of the spiral ganglion in the rats with DM compared to the normal animals $(P<0.001)$ (Fig. 4A). The total volume of spiral ganglion of the groups of DM+R50, DM+R100, and DM+R150 was larger by $27.44 \%, 36.11 \%$, and $44.21 \%$, respectively, than that of the DM animals $(P<0.05)$ (Fig. 4A).
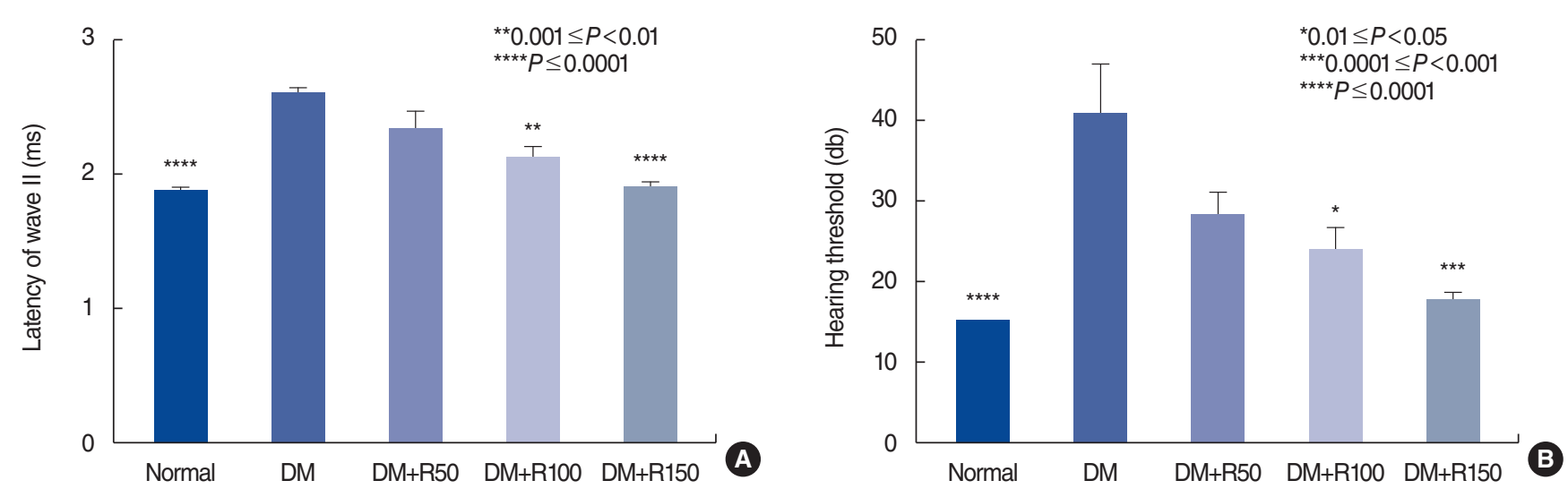

Fig. 3. (A) Latency of wave II in normal, diabetes mellitus (DM), and rutin-treated groups. (B) Hearing threshold in the normal, DM, and rutintreated groups. All comparisons are with the DM group. R50, $50 \mathrm{mg} / \mathrm{kg}$ of rutin; R100, $100 \mathrm{mg} / \mathrm{kg}$ of rutin; R150, $150 \mathrm{mg} / \mathrm{kg}$ of rutin. 


\section{Spiral ganglion neuron count}

The number of spiral ganglion neurons was $52.90 \%$ lower in the DM group than in the normal rats $(P<0.001)$ (Fig. 4B). The total number of spiral ganglion neurons was $29.38 \%, 39.29 \%$, and $53.68 \%$ higher in the DM rats that had received 50, 100, and

Table 1. Different forms of wave $\mathrm{V}$ in the normal, DM, and rutin-treated groups

\begin{tabular}{lcccc}
\hline Group & $\begin{array}{c}\text { Normal V } \\
\text { wave (\%) }\end{array}$ & $\begin{array}{c}\text { Delay V } \\
\text { wave (\%) }\end{array}$ & $\begin{array}{c}\text { Absent V } \\
\text { wave (\%) }\end{array}$ & P-value \\
\hline Normal & 100 & 0 & 0 & $<0.0001$ \\
DM & 0 & 0 & 100 & - \\
DM+R50 & 25 & 12.5 & 62.5 & 0.99 \\
DM+R100 & 12.5 & 50 & 37.5 & 0.701 \\
DM+R150 & 25 & 75 & 0 & 0.026 \\
\hline
\end{tabular}

All comparisons are with the DM group.

DM, diabetes mellitus; R50, $50 \mathrm{mg} / \mathrm{kg}$ of rutin; R100, $100 \mathrm{mg} / \mathrm{kg}$ of rutin; R150, $150 \mathrm{mg} / \mathrm{kg}$ of rutin.

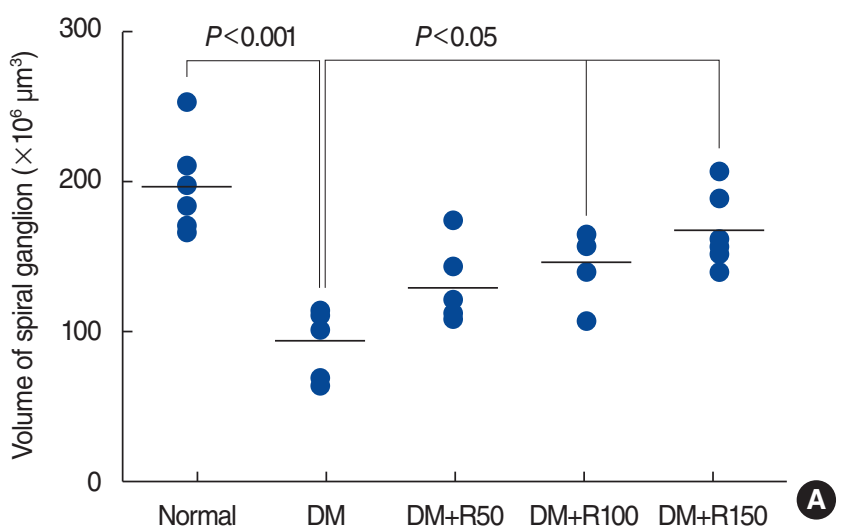

$150 \mathrm{mg} / \mathrm{kg}$ of rutin, respectively, than in the DM group $(P<0.05)$ (Fig. 4B).

\section{Biochemical assays}

Rutin improved oxidative stress markers in plasma. Our results also indicated that rutin treatment restored the antioxidant activity of SOD $(P<0.05)$ (Fig. 5A) and significantly reduced plasma levels of MDA, an oxidative marker $(P<0.05$ and $P<0.001$, respectively) (Fig. 5B).

\section{DISCUSSION}

Our results indicate that rutin had a significant therapeutic effect on diabetic auditory neuropathy in rats. Rutin is under investigation as a drug with antioxidant activity that might be able to prevent and treat diabetic neuropathy. In our study, rutin was significantly effective at therapeutic doses of $100 \mathrm{mg} / \mathrm{kg}$ and

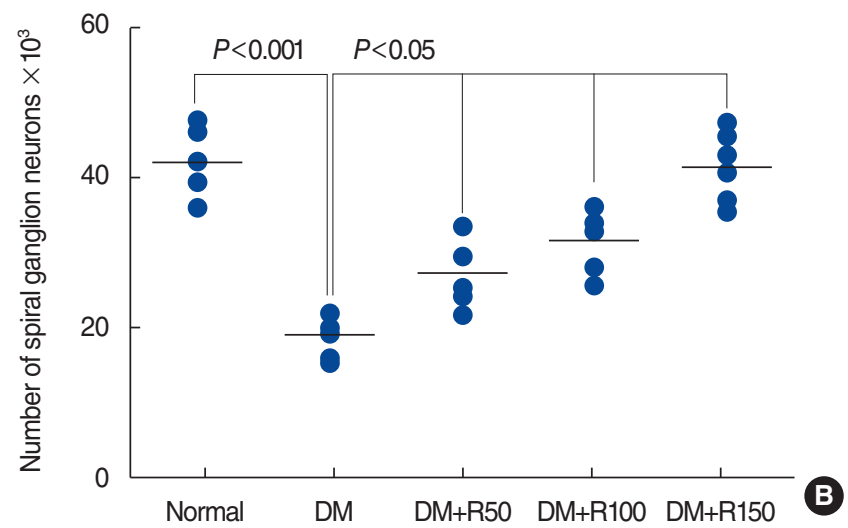

Fig. 4. Aligned dot plots of the volumes of the spinal ganglion $(A)$ and the number of spiral ganglion neurons (B) in the normal, DM, DM+R50, $\mathrm{DM}+\mathrm{R} 100$, and DM+RF150 groups. Each dot indicates an animal and the horizontal bar is the mean of the corresponding parameter. Significant differences and $P$-values have been indicated on each plot. All comparisons are with the DM group. DM, diabetes mellitus; R50, $50 \mathrm{mg} / \mathrm{kg}$ of rutin; R100, $100 \mathrm{mg} / \mathrm{kg}$ of rutin; R150, $150 \mathrm{mg} / \mathrm{kg}$ of rutin.
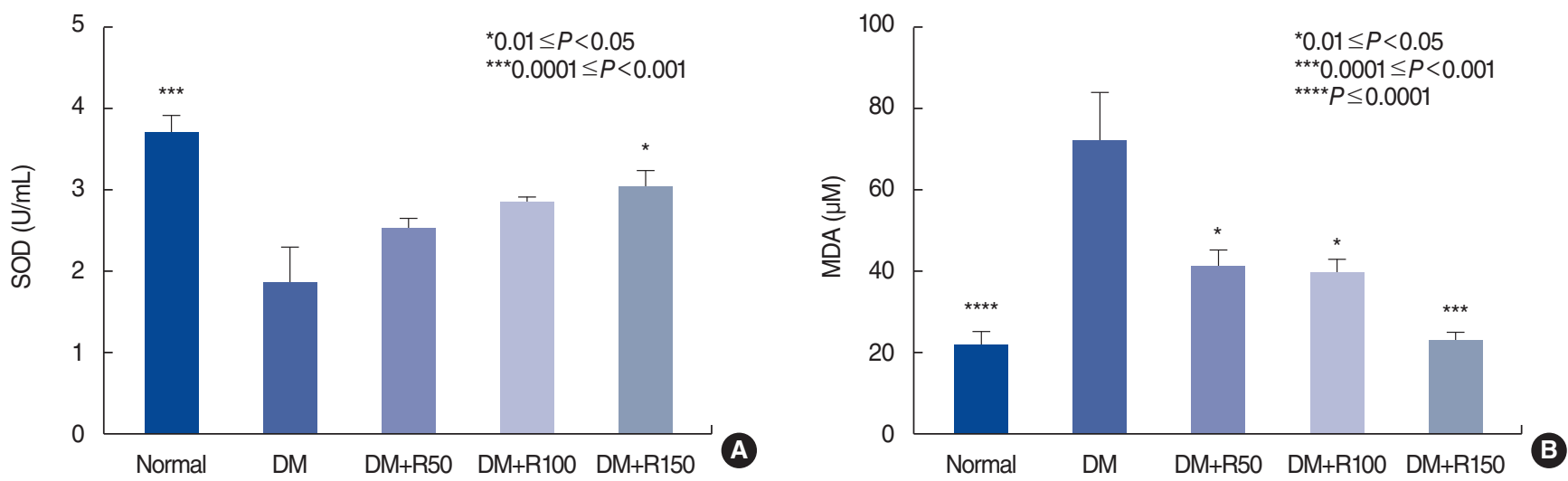

Fig. 5. Plasma concentration of superoxide dismutase (SOD; an antioxidant marker, A) and malondialdehyde (MDA; an oxidative marker, B) in the normal, diabetes mellitus, and rutin-treated groups. All comparisons are with the DM group. DM, diabetes mellitus; R50, 50 mg/kg of rutin; $\mathrm{R} 100,100 \mathrm{mg} / \mathrm{kg}$ of rutin; R150, $150 \mathrm{mg} / \mathrm{kg}$ of rutin. 
$150 \mathrm{mg} / \mathrm{kg}$. At a dose of $50 \mathrm{mg} / \mathrm{kg}$, we observed improvement of auditory neuropathy, but not to a statistically significant extent for ABR recordings.

In our study, the effect of rutin on diabetic auditory neuropathy was evaluated by ABR recordings, spiral ganglion stereology, and measurements of SOD and MDA in plasma. These evaluation tools, especially the ABR recordings and stereological studies, provided more accurate and integrative information about diabetic auditory dysfunction and morphological changes than has been reported in previous studies of diabetic neuropathy and its treatment. ABR is a valuable tool for evaluating auditory neural function. It has been used to assess the induction and treatment of auditory neuropathy in many experimental studies. There are some differences between human and rats in ABR parameters and morphology that we considered in our study. Many authors have stated that wave II is the most prominent in rats, whereas wave $\mathrm{V}$ is most prominent in humans. We evaluated parameters such as latency and threshold for waves II andV. It seems that wave II is generated in the cochlear nucleus complex in rats. This wave involves more electrical activity and may be analogous to wave III in humans. We found that DM caused elevation of the ABR threshold ( $40 \pm 5 \mathrm{~dB}$ SPL). Other clinical and experimental studies have also shown similar effects of diabetes on the hearing threshold. Increased hearing threshold and latency, with changes in wave forms, have been detected in patients with diabetes during ABR recordings $[7,33,34]$. An experimental study showed that the hearing threshold was elevated 5 weeks after diabetes induction in mice. Increased latency, especially of waves I and IV, was another notable finding in the ABR recordings in that study [35]. ABR changes in diabetic mice were detected at 2-10 weeks following STZ injection. Elevated auditory thresholds and delays in wave formation (waves I, II, III, and IV) were the main features of ABR changes in an animal study [7]. In humans, an elevated auditory threshold and high-frequency hearing loss were detected in patients with diabetic nephropathy. However, no significant correlation between the duration of diabetes and degree of hearing loss was found [36].

After 8 weeks of rutin treatment (at $150 \mathrm{mg} / \mathrm{kg} /$ day), the ABR threshold measurement showed almost normal values $(20 \pm 5 \mathrm{~dB}$ SPL). DM significantly increased wave II latency $(2.6 \pm 0.1 \mathrm{~ms})$. Rutin treatment, especially at a dose of $150 \mathrm{mg} / \mathrm{kg}$, prevented this delay, yielding values that were close to normal $(1.9 \pm 0.1 \mathrm{~ms})$. For wave $\mathrm{V}$, we merely focused on its presence, since the variations in other studies led us to conclude that latency or amplitude measurements of wave $\mathrm{V}$ might not be accurate. Our study showed significant improvements in wave $\mathrm{V}$ in rutin-treated diabetic rats.

In a previous study, rutin successfully prevented sciatic neuropathy in diabetic rats [16]. We observed that a dose of $150 \mathrm{mg} / \mathrm{kg}$ was more effective than a dose of $100 \mathrm{mg} / \mathrm{kg}$, which is line with other findings stating that the response to rutin is dose-dependent $[16,37]$. In diabetic rats, rutin has shown significant results in terms of controlling hyperglycemia, hyperlipidemia, liver glycogen content, and oxidative stress [38]. Rutin is believed to increase conduction velocity in sensory and motor nerves. This effect was shown in a rat experimental study with a dose of $50 \mathrm{mg} / \mathrm{kg}$. A slight response was demonstrated at lower doses of $5 \mathrm{mg} / \mathrm{kg}$ and $25 \mathrm{mg} / \mathrm{kg}$ [39]. These findings also reinforce the dose-dependent responses of rutin that we also observed in our study. At a higher dose $(200 \mathrm{mg} / \mathrm{kg})$, rutin was able to regenerate the myelin sheath in damaged sciatic nerves in a diabetic rat model [15].

The quantitative analysis of histology in the stereological method that we used enabled a more reliable assessment of structural changes following treatment. The stereological evaluation in our study showed significant decreases in the total volume and number of neurons in the spiral ganglia of diabetic rats. Other studies have likewise shown structural changes in the spiral ganglion in DM, but a notable aspect of our study is that we used the stereological method, which is considered to be more accurate for detecting cell loss. We found that rutin significantly prevented the decrease of volume and neuronal loss in the spiral ganglion. This finding highlights the antioxidant and neuroprotective functions of rutin. Similar to ABR recordings, the stereological examination showed better responses to rutin at a dose of $150 \mathrm{mg} / \mathrm{kg}$ than at doses of $100 \mathrm{mg} / \mathrm{kg}$ and $50 \mathrm{mg} / \mathrm{kg}$, again reflecting the dose-dependent action of this drug.

Lee et al. [40] performed a similar study in diabetic mice, in which they investigated spiral ganglion morphology. They used ABR recordings to detect neural dysfunction and scanning electron microscopy for histological examinations, and concluded that DM causes early degeneration of spiral ganglion cells. Some studies have reported morphological changes in diabetic auditory neuropathy. These changes consist of thickening of the basilar membrane in the stria vascularis, atrophy of the stria vascularis, outer and inner hair cell loss, and loss of neurons in the spiral ganglion [6].

We used SOD measurements as a tool to evaluate the effects of rutin on auditory neuropathy in diabetic rats. The restoration of SOD levels after neuropathy treatment has been investigated in many studies $[15,16,18]$. Our results also indicate that rutin ameliorates auditory neuropathy by normalizing SOD. Since the effects of rutin are dose-dependent, SOD levels returned to values closer to normal in the $150 \mathrm{mg} / \mathrm{kg}$ group than in the $100 \mathrm{mg} / \mathrm{kg}$ group. The reduction of MDA levels by rutin treatment furnishes evidence in support of this novel treatment for diabetic auditory neuropathy. MDA levels have been evaluated as an oxidative marker in some experimental and clinical trials of antioxidant agents, with relatively successful results [15,16,18].

The treatment and control of diabetic auditory neuropathy have a major effect on patients' quality of life. For this purpose, several drugs have been investigated. This study revealed that rutin was effective in preventing auditory diabetic neuropathy in a murine model. However, further clinical trials are warranted to evaluate the efficacy of this drug in humans. 


\section{CONFLICT OF INTEREST}

No potential conflict of interest relevant to this article was reported.

\section{ACKNOWLEDGMENTS}

The present article was extracted from the thesis written by Aida Doostkam and was financially supported by Shiraz University of Medical Sciences (grant No. 96-16690).

The authors thank Dr. Afsaneh Doosti for technical assistance. We also wish to thank Mr. H. Argasi at the Research Consultation Center of Shiraz University of Medical Sciences for his invaluable assistance in editing this manuscript.

\section{ORCID}

$\begin{array}{ll}\text { Aida Doostkam } & \text { https://orcid.org/0000-0003-3348-573X } \\ \text { Hossein Mirkhani } & \text { https://orcid.org/0000-0002-7433-1174 } \\ \text { Kamyar Iravani } & \text { https://orcid.org/0000-0001-8302-2683 } \\ \text { Saied Karbalay-Doust } & \text { https://orcid.org/0000-0003-1071-1730 } \\ \text { Kazem Zarei } & \text { https://orcid.org/0000-0002-6585-2509 }\end{array}$

\section{AUTHOR CONTRIBUTIONS}

Conceptualization: HM, KI, SKD. Data curation: AD, KI, SKD, KZ. Formal analysis: AD. Funding acquisition: HM. Methodology: AD. Project administration: HM, KI. Visualization: AD, HM, KI. Writing-original draft: KI. Writing-review \& editing: AD, $\mathrm{HM}, \mathrm{KI}, \mathrm{SKD}$

\section{REFERENCES}

1. Tousoulis D, Papageorgiou N,Androulakis E, Siasos G, Latsios G,Tentolouris $\mathrm{K}$, et al. Diabetes mellitus-associated vascular impairment: novel circulating biomarkers and therapeutic approaches. J Am Coll Cardiol. 2013 Aug;62(8):667-76.

2. Kampoli AM, Tousoulis D, Briasoulis A, Latsios G, Papageorgiou N, Stefanadis C. Potential pathogenic inflammatory mechanisms of endothelial dysfunction induced by type 2 diabetes mellitus. Curr Pharm Des. 2011 Dec;17(37):4147-58.

3. Asmat U,Abad K, Ismail K. Diabetes mellitus and oxidative stress-A concise review. Saudi Pharm J. 2016 Sep;24(5):547-53.

4. Feldman EL, Nave KA, JensenTS, Bennett DL. New horizons in diabetic neuropathy: mechanisms, bioenergetics, and pain. Neuron. 2017 Mar;93(6):1296-313.

5. Ozougwu JC, Obimba KC, Belonwu CD, Unakalamba CB. The pathogenesis and pathophysiology of type 1 and type 2 diabetes mellitus. J Physiol Pathophysiol. 2013;4(4):46-57.

6. Akinpelu OV, Ibrahim F, Waissbluth S, Daniel SJ. Histopathologic changes in the cochlea associated with diabetes mellitus: a review.
Otol Neurotol. 2014;35(5):764-74.

7. Hong BN, Kang TH.Auditory neuropathy in streptozotocin-induced diabetic mouse. Neurosci Lett. 2008 Feb;431(3):268-72.

8. Varkonyi T, Korei A, Putz Z, Martos T, Keresztes K, Lengyel C, et al. Advances in the management of diabetic neuropathy. Minerva Med. 2017 Oct;108(5):419-37.

9. Hosseini A, Abdollahi M. Diabetic neuropathy and oxidative stress: therapeutic perspectives. Oxid Med Cell Longev. 2013;2013:168039.

10. Han Y, Wang M, Shen J, Zhang Z, Zhao M, Huang J, et al. Differential efficacy of methylcobalamin and alpha-lipoic acid treatment on symptoms of diabetic peripheral neuropathy. Minerva Endocrinol. 2018 Mar;43(1):11-8.

11. Yilmaz M,Aktug H, Oltulu F, Erbas O. Neuroprotective effects of folic acid on experimental diabetic peripheral neuropathy. Toxicol Ind Health. 2016 May;32(5):832-40.

12. Ganeshpurkar A, Saluja AK. The pharmacological potential of rutin. Saudi Pharm J. 2017 Feb;25(2):149-64.

13. Ghorbani A. Mechanisms of antidiabetic effects of flavonoid rutin. Biomed Pharmacother. 2017 Dec;96:305-12.

14. Habtemariam S, Lentini G. The therapeutic potential of rutin for diabetes: an update. Mini Rev Med Chem. 2015;15(7):524-8.

15. Mittal R, Kumar A, Singh DP, Bishnoi M, Nag TC. Ameliorative potential of rutin in combination with nimesulide in STZ model of diabetic neuropathy: targeting Nrf2/HO-1/NF-kB and COX signalling pathway. Inflammopharmacology. 2018 Jun;26(3):755-68.

16. Niture NT, Patil DG, Somani RS, Sahane RS. Effect of rutin on early diabetic neuropathy in experimental animals. J Nat Prod Plant Resour. 2014;4(4):1-9.

17. Xie L,Wang M, LiaoT, Tan S, Sun K, Li H, et al.The characterization of auditory brainstem response (ABR) waveforms: a study in tree shrews (Tupaia belangeri). J Otol. 2018 Sep;13(3):85-91.

18. Norrix LW,Velenovsky D. Unraveling the mystery of auditory brainstem response corrections: the need for universal standards. J Am Acad Audiol. 2017 Nov/Dec;28(10):950-60.

19. Helzner EP, Contrera KJ. Type 2 diabetes and hearing impairment. Curr Diab Rep. 2016 Jan;16(1):3.

20. Wang Y, Branicky R, Noe A, Hekimi S. Superoxide dismutases: dual roles in controlling ROS damage and regulating ROS signaling. J Cell Biol. 2018 Jun;217(6):1915-28.

21. Younus H.Therapeutic potentials of superoxide dismutase. Int J Health Sci (Qassim). 2018 May-Jun;12(3):88-93.

22. Islam MT. Oxidative stress and mitochondrial dysfunction-linked neurodegenerative disorders. Neurol Res. 2017 Jan;39(1):73-82.

23. Bouhajja H, Kacem FH, Abdelhedi R, Ncir M, Dimitrov JD, Marrakchi $\mathrm{R}$, et al. Potential predictive role of lipid peroxidation markers for type 2 diabetes in the adult Tunisian population. Can J Diabetes. 2018 Jun;42(3):263-71.

24. Abeeleh MA, Ismail ZA,Alzaben KR,Abu-Halaweh SA,Al-Essa MK, Abuabeeleh $\mathrm{J}$, et al. Induction of diabetes mellitus in rats using intraperitoneal streptozotocin: a comparison between 2 strains of rats. Eur J Sci Res. 2009;32(3):398-402.

25. Hernandez-Aquino E, Zarco N, Casas-Grajales S, Ramos-Tovar E, Flores-Beltran RE, Arauz J, et al. Naringenin prevents experimental liver fibrosis by blocking TGFB-Smad3 and JNK-Smad3 pathways. World J Gastroenterol. 2017 Jun;23(24):4354-68.

26. Kuse H, Ogawa T, Nakamura N, Nakayama Y, Nakakarumai A, Komori $\mathrm{C}$, et al. Changes in auditory brainstem response (ABR) in Kanamycin-induced auditory disturbance model rats. JToxicol Sci. 2011; 36(6):835-41.

27. Marquez-Gamino S, Sotelo F, Sosa M, Caudillo C, Holguin G, Ramos $\mathrm{M}$, et al. Pulsed electromagnetic fields induced femoral metaphyseal bone thickness changes in the rat. Bioelectromagnetics. $2008 \mathrm{Jul}$; 29(5):406-9.

28. Rusznak Z, Szucs G. Spiral ganglion neurones: an overview of mor- 
phology, firing behaviour, ionic channels and function. Pflugers Arch. 2009 Apr;457(6):1303-25.

29. Kristiansen SL, Nyengaard JR. Digital stereology in neuropathology. APMIS. 2012 Apr;120(4):327-40.

30. Schettino AE, Lauer AM. The efficiency of design-based stereology in estimating spiral ganglion populations in mice. Hear Res. 2013 Oct;304:153-8.

31. Gundersen HJ, Bagger P, Bendtsen TF, Evans SM, Korbo L, Marcussen N, et al. The new stereological tools: disector, fractionator, nucleator and point sampled intercepts and their use in pathological research and diagnosis. APMIS. 1988 Oct;96(10):857-81.

32. Gundersen HJ, BendtsenTF, Korbo L, Marcussen N, Moller A, Nielsen $\mathrm{K}$, et al. Some new, simple and efficient stereological methods and their use in pathological research and diagnosis. APMIS. 1988 May; 96(5):379-94.

33. Radwan HM, El-Gharib AM, Erfan AA, Emara AA. Auditory brain stem response and cortical evoked potentials in children with type 1 diabetes mellitus. Acta Otolaryngol. 2017 May;137(5):511-5.

34. Tsuda J, Sugahara K, Hori T, Kanagawa E, Takaki E, Fujimoto M, et al. A study of hearing function and histopathologic changes in the cochlea of the type 2 diabetes model Tsumura Suzuki obese diabetes mouse.Acta Otolaryngol. 2016 Nov;136(11):1097-106.
35. Hong BN, Kang TH. Distinction between auditory electrophysiological responses in type 1 and type 2 diabetic animal models. Neurosci Lett. 2014 Apr;566:309-14.

36. Ren J, Ma F, ZhouY, Xu A, Zhang J, Ma R, et al. Hearing impairment in type 2 diabetics and patients with early diabetic nephropathy. $\mathrm{J}$ Diabetes Complications. 2018 Jun;32(6):575-9.

37. Hosseinzadeh H, Nassiri-Asl M. Review of the protective effects of rutin on the metabolic function as an important dietary flavonoid. J Endocrinol Invest. 2014 Sep;37(9):783-8.

38. Ahmed OM, Moneim AA, Yazid IA, Mahmoud AM. Antihyperglycemic, antihyperlipidemic and antioxidant effects and the probable mechanisms of action of Ruta graveolens infusion and rutin in nicotinamide-streptozotocin-induced diabetic rats. Diabetol Croat. 2010; 39(1):15-35.

39. Tian R, Yang W, Xue Q, Gao L, Huo J, Ren D, et al. Rutin ameliorates diabetic neuropathy by lowering plasma glucose and decreasing oxidative stress via Nrf2 signaling pathway in rats. Eur J Pharmacol. 2016 Jan;771:84-92.

40. Lee HS, Kim KR, Chung WH, Cho YS, Hong SH. Early sensorineural hearing loss in ob/ob mouse, an animal model of type 2 diabetes. Clin Exp Otorhinolaryngol. 2008 Dec;1(4):211-6. 